\title{
GENERALIZED TOTALLY DISCONNECTEDNESS
}

\author{
CHARLES DORSETT
}

Department of Mathematıcs and Statıstıcs

Louısıana Tcch University

Ruston, Loussiana

(Received June 15, 1993 and in revised form March 22, 1994)

ABSTR $\Lambda$ C'T In thıs paper totally disconnectedness is generalızed to maxımal disconnectedness, which is investigated, and additional propertıes of totally disconnectedness and 0-dımensıonal are given.

KEY WORDS AND PIIRASFS Totally disconncctedness, 0-dimensional, and $T_{0}$ - identification spaccs 1992 AMS SUBJECT CIASSIFICATION CODE. 54A05, 54D05

\section{INTRODUCTION}

Totally disconnected spaces were considered as early as 1921 by Knaster and Kuratowski [1] and by Sierpinski [2]. A space $(X, T)$ is totally disconnected iff the components of $(X, T)$ are the points. Qucstions about possible gencralızatıons of totally disconnected spaces led to the following discovery.

TIIEOREM 1. Let $(X, T)$ be a totally disconnected spacc. Then $(X, T)$ is $T_{1}$.

PROOF: Since components are closed, then the singleton sets are closed, which implies $(X, T)$ is $T_{1}$.

Thus for $T_{1}$ spaces, totally disconnectedness is maximal disconnectedness in the sense that the components are the smallest possible sets, which motıvated the introduction and investigation of maximal disconnected spaces in this paper.

\section{MAXIMAL DISCONNECTEDNESS.}

DEFINITION 1. Let $(X, T)$ be a space and let $C$ be a component of $(X, T)$. Then $C$ is a minimal compon nnt of $(X, T)$ iff $C$ does not contain a nonempty proper closed connected subset. The space $(X, T)$ is maximal disconnected iff the components of $(X, T)$ are minimal components.

Note that for a space $(X, T), C l(\{x\})$ is connected for each $x \in X$ and that a component $C$ of $(X, T)$ is a minimal component of $(X, T)$ iff $C=C l(\{x\})$ for each $x \in X$.

In $1961 \mathrm{~A}$. Davis [3] was interested in properties $R_{\mathfrak{i}-1}$ weaker than $T_{\mathfrak{i}}$, which together with $T_{\mathfrak{i}-1}$, would be equivalent to $T_{3}, i=1,2$. In the 1961 investigation $R_{0}$ and $R_{1}$ spaces were defined. A space $(X, T)$ is $R_{0}$ iff one of the following equivalent conditions is satisfied: (a) if $O \in T$ and $x \in O$, then $C l(\{x\}) \subset O$, and (b) $\{C l(\{x\}) \mid x \in X\}$ is a decomposition of $X$. A space $(X, T)$ is $R_{1}$ iff for $x, y \in X$ such that $C l(\{x\}) \neq C l(\{y\})$, there exist disjoint open sets $U$ and $V$ such that $C l(\{x\}) \subset U$ and $C l(\{y\}) \subset V$. The 1961 paper [3] was a continuation of work done by $\mathrm{N}$. Shanin in 1943 [4], in which $R_{0}$ spaces were called weak regular spaces. Combining this information with the note above in a straightforward proof, which is omitted, gives the following result.

THEOREM 2. Let $(X, T)$ be a space. Then $(X, T)$ is maxımal disconnected iff $(X, T)$ is $R_{0}$ and the components of $(X, T)$ are closures of singleton sets.

The results above can be combined to obtain the following result.

COROLLARY 3 . Let $(X, T)$ be a space. Then the following are equivalent: (a) $(X, T)$ is totally disconnccted, 
(b) $(\mathrm{Y}, T)$ is $T_{1}$ maximal disconnerted, and (c) $(\mathrm{K}, T)$ is $T_{0}$ maximal disconnerted

TIIEORCM 4. Let $(X, T)$ be a space Then $(Y, T)$ is maximal disconnected iff every homeomorphic image of $(\mathrm{Y}, T)$ is maximal disconnecird.

The straightforward proof is omıtted

('ombining Theorem 4 with C'orollary 3 and the fart that $T_{1}$ is a topologiral property gives the following result

(OROLLARY 5) Totally disconnectedness is a topological property.

In 1977 [5] $T_{0}-\imath d e n t i f i c a t i o n$ spaces were used to furtlice investigate and better understand $R_{0}$ spaces Let $(X, T)$ be a space and let $R$ be the equivalence relation on $X$ defined by $x R y$ iff $C l(\{x\})=C l(\{y\})$ Then the $T_{0}$ - idcntıfication space of $(X, T)$ is $(X(T O), Q(T O))$, where $X(T O)$ is the set of equivalence classes of $R$ and $Q(T O)$ is the decomposition topology on $\mathrm{X}(T O)[6]$ The space $(X, T)$ is $R_{0}$ iff $(X(T O), Q(T O))$ is $T_{1}$ [6]. Lct. $P(T O) \quad(X, T) \rightarrow(X(T O), Q(T O))$ be the natural map. Below, $T_{0}-i d c n t i f i c a t i o n$ spaces are used to furtlicr investıgate maximal disconnected spaces

TIIEOREM 6. Let $(X, T)$ be a space. Then $(X, T)$ is maximal disconnected iff $(X(T O), Q(T O))$ is totally disconnected

PROOF Suppose $(X, T)$ is maxımal dısconnected. Let $\mathcal{C}$ be a component of $(X(T O), Q(T O))$. Since $(X, T)$ Is $R_{0}$, then $X(T O)=\{C l(\{x\}) \mid x \in X\}$ [5]. Let $C l(\{x\}) \in \mathcal{C}$. Then $P(T O)^{-1}(\mathcal{C})$ is a closed and connected [7] subset of $(X, T)$ containıng $x$, which implıes $P(T O)^{-1}(\mathcal{C})=C l(\{x\})$ and $\mathcal{C}=\{C l(\{x\})\}$. Thus components of $(X(T O), Q(T O))$ are singleton sets, which implies $(X(T O), Q(T O))$ is totally disconnected.

Converscly, suppose $(X(T O), Q(T O))$ is totally disconnected. Let $C$ be a component of $(X, T)$. Let $x \in$ C. Since $(X(T O), Q(T O))$ is totally disconnected, then $(X(T O), Q(T O))$ is $T_{1}$, which implies $(X, T)$ is $R_{0}$ and $X(T O)=\{C l(\{x\}) \mid x \in X\}$. Since $P(T O)$ is continuous and closed [7], then $P(T O)(C)$ is closed connected, with $C l(\{x\}) \in P(T O)(C)$, which implies $P(T O)(C)=\{C l(\{x\})\}$ and thus $C=C l(\{x\})$. Hence $(X, T)$ is maximal disconnected.

Combining the results above with the fact that for a space $(X, T),(X(T O), Q(T O))$ is $T_{0}$ gives the following result.

COROLLARY 7. Let $(X, T)$ be a space. Then $(X, T)$ is maximal disconnected iff $(X(T O), Q(T O))$ is maximal disconnected.

TIIEOREM 8. Let $(X, T)$ be a space, let $Y \subset X$, and let $T_{Y}$ be the relative $T$ topology on $Y$. Then $\left(Y\left(T_{Y} O\right), Q\left(T_{Y} O\right)\right)$ is homeomorphic to $\left(P(T O)(Y), Q(T O)_{P(T O)(Y)}\right)$.

PROOF. For each $y \in Y$ let $K_{y} \in Y\left(T_{Y} O\right)$ containing $y$ and let $C_{y} \in P(T O)(Y)$ containing $y$. Let $f=$ $\left\{\left(K_{y}, C_{y}\right) \mid y \in Y\right\}$. If $K_{y}=K_{z}$, then $C l_{T_{Y}}(\{y\})=C l_{T_{Y}}(\{z\})$, which implies $C l_{T}(\{y\})=C l_{T}(\{z\})$ and $C_{y}=C_{z}$. Thus $f$ is a function. Clearly $f$ is onto. If $C_{y}=C_{z}$, where $y, z \in Y$, then $C l_{T}(\{y\})=C l_{T}(\{z\})$, which implies $C l_{T_{Y}}(\{y\})=C l_{T_{Y}}(\{z\})$ and $K_{y}=K_{z}$. Thus $f$ is one-to-one. Let $\mathcal{O} \in Q(T O)_{P(T O)(Y)}$. Let $\mathcal{U} \in Q(T O)$ such that $\mathcal{O}=\mathcal{U} \cap P(T O)(Y)$. Then $f^{-1}(\mathcal{O})=P\left(T_{Y} O\right)\left(P(T O)^{-1}(\mathcal{U}) \cap Y\right)$ and since $P(T O)^{-1}(\mathcal{U}) \cap Y \in T_{Y}$ and $P\left(T_{Y} O\right)$ is open [7],

then $f^{-1}(\mathcal{O}) \in Q\left(T_{Y} O\right)$. Thus $f$ is continuous. If $\mathcal{V} \in Q\left(T_{Y} O\right)$, then $P\left(T_{Y} O\right)^{-1}(\mathcal{V})=W \cap Y$, where $W \in T$,

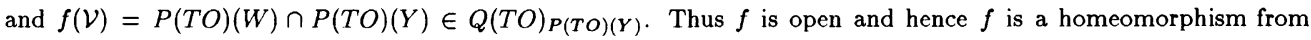
$\left(Y\left(T_{Y} O\right), Q\left(T_{Y} O\right)\right)$ onto $\left(P(T O)(Y), Q(T O)_{P(T O)(Y)}\right)$.

THEOREM 9. Let $(X, T)$ be a space. Then $(X, T)$ is maximal disconnected iff every subspace of $(X, T)$ is maximal disconnected.

PROOF: Suppose $(X, T)$ is maximal disconnected. Let $Y \subset X$. Then $(X(T O), Q(T O))$ is totally disconnected, which implies $\left(P(T O)(Y), Q(T O)_{P(T O)(Y)}\right)$ is totally disconnected $[6]$. Since $\left(P(T O)(Y), Q(T O)_{P(T O)(Y)}\right)$ is homeomorphic to $\left(Y\left(T_{Y} O\right), Q\left(T_{Y} O\right)\right)$, then $\left(Y\left(T_{Y} O\right), Q\left(T_{Y} O\right)\right)$ is totally disconnected, which implies $\left(Y, T_{Y}\right)$ is maximal disconnected

Clearly, the converse is true. 
TIICOREM 10 Let $\left\{\left(Y_{\alpha}, T_{\alpha}\right) \mid \alpha \in A\right\}$ be a nonemply collection of nonempty spaces. Then $\left(Y_{\alpha}, T_{\alpha}\right)$ is maximal disconnected for rarh $\alpha \in A$ iff $\left(\prod_{n \in A}, X_{\alpha} . W^{\circ}\right)$ is maximal disconnected, where $W^{*}$ is the product topology on $\prod_{x \in A} \cdot V_{r}$

PROOI Suppose $\left(\mathrm{K}_{\alpha}, T_{\alpha}\right)$ is maximal disconnected for carh $\alpha \in A$ Then $\left(\mathrm{Y}_{\alpha}\left(T_{\alpha} O\right), Q\left(T_{\alpha} O\right)\right)$ is tolally disconnected for each $\alpha \in A$ and $\left(\prod_{\alpha \in A} x_{\alpha}\left(T_{\Omega} O\right)\right.$, $\left.\mathcal{W}\right)$, where $\mathcal{W}$ is llic product topology on $\prod_{\alpha \in A} x_{\Omega}\left(T_{\Omega} O\right)$. is totally disconnected [6] Since $\left(\prod_{\alpha \in A}, X_{\alpha}\left(T_{\alpha} O\right), \mathcal{W}\right)$ is homeomorphic to $\left(\left(\prod_{\alpha \in A} X_{\alpha}\right)(H O), Q(H O)\right)[8]$, Ihen $\left(\left(\prod_{\alpha \in A} X_{\alpha}\right)(W O), Q(W O)\right)$ is totally disconnected, which implics $\left(\prod_{\alpha \in 1}, X_{\alpha}, W\right)$ is maximal disconncetced.

Conversely, suppose $\left(\prod_{\alpha \in A} X_{\alpha}, W\right)$ is maximal disconnected Let $\beta \in A$. Let $C_{\beta}$ be a component of $\left(X_{\beta}, T_{\beta}\right)$. Let $x_{3} \in C_{\beta}$ For each $\alpha \in A, \alpha \neq \beta$, let $C_{\alpha}$ be a component of $\left(X_{\alpha}, T_{\alpha}\right)$ and let $x_{\alpha} \in C_{\alpha}$. Then $\prod_{\alpha \in A} C_{\alpha}$ is a closed connerted subsel of $\prod_{\alpha \in A} X_{\alpha}$ containıng $x=\prod_{\alpha \in A}\left\{x_{\alpha}\right\}$, whıch implıes $\prod_{\alpha \in A} C_{\alpha}^{\prime}=C l(\{x\})=\prod_{\alpha \in A} C l\left(\left\{x_{\alpha}\right\}\right)$ and $C_{B}=C l\left(\left\{x_{B}\right\}\right)$ Thus $\left(X_{3}, T_{\beta}\right)$ is maxımal disconnected

Combining the results above with the fact that the product space of a nonempty collection of nonempty spaces is $T_{1}$ iff rach factor space is $T_{1}$ gives the next result.

C'OROLLARY 11 Let $\left\{\left(X_{\alpha}, T_{\alpha}\right) \mid \alpha \in A\right\}$ be a nonempty collection of nonempty spaces. Then $\left(X_{\alpha} \cdot T_{\alpha}\right)$ is lotally disconnected for each $\alpha \in A$ iff $\left(\prod_{\alpha \in A} X_{\alpha}, W\right)$ is totally disconnected.

In S. Wıllard's 1970 book [6] relationships between totally disconnected and 0-dimensıonal were examıned. $\Lambda$ space $(X, T)$ is 0-dimensional iff each point of $X$ has a neighborhood base consistıng of closed open sets. Below, results in Willard's book are used not only to further investıgatc maxımal disconnectedness, but also, to further investıgate totally disconncctedness.

Since every 0-dimensional space is completely regular, then every 0-dimensional space is regular, and thus $R_{1}$ $[9]$ and $R_{0}[3]$.

TIIEOREM 12. Let $(X, T)$ be a space. Then $(X, T)$ is 0 -dimensional iff $(X(T O), Q(T O))$ is 0-dimensional.

PROOF: Suppose $(X, T)$ is 0-dimensional. Let $C \in X(T O)$. Let $x \in C$. Let $\mathcal{O}$ be a neighborhood base of $x$ consistıng of closed open sets. Since $P(T O)$ is closed, open, and continuous [7], then $P(T O)(\mathcal{O})$ is a ncighborhood base of $C$ consisting of closed open sets. Thus (X(TO), $Q(T O))$ is 0-dimensional.

Converscly, suppose $(X(T O), Q(T O))$ is 0 -dimensional. Let $x \in X$. Let $C \in X(T O)$ such that $x \in C$. Let $\mathcal{O}$ be a neighborhood base of $C$ consisting of closed open sets. Since

$P(T O)^{-1}(P(T O)(O))=O$ for each $O \in T[7], P(T O)^{-1}(\mathcal{O})$ is a neighborhood base of $x$ consisting of closed open sets. Thus $(X, T)$ is 0 -dimensional.

THEOREM 13. Let $(X, T)$ be a 0 -dimensional space. Then $(X, T)$ is maximal disconnected.

PROOF. Since $(X, T)$ is 0 -dimensional, then $(X, T)$ is $R_{0}$ and $(X(T O), Q(T O))$ is 0 -dimensional $T_{1}$, which implies $(X(T O), Q(T O))$ is totally disconnected $[6]$ and $(X, T)$ is maximal disconnected.

COROLLARY 14 . Every 0-dimensional $T_{0}$ space is totally disconnected.

DEFINITION 2. A space is rim-compact iff each of its points has a base of neighborhoods with compact frontier $[6]$.

THEOREM 15. Let $(X, T)$ be a space. Then $(X, T)$ is rim-compact iff $(X(T O), Q(T O))$ is rim-compact.

PROOF: Suppose $(X, T)$ is rim-compact. Let $C \in X(T O)$. Let $x \in C$. Let $\mathcal{O}$ be a neighborhood base of $x$ consisting of neighborhoods with compact frontiers. Let $O \in \mathcal{O}$. Then $\operatorname{Fr}(\operatorname{Int}(O))$ is a closed subset of the compact set $\operatorname{Fr}(O)$, which implies $\operatorname{Fr}(\operatorname{Int}(O))$ is compact. Then $P(T O)(\operatorname{Int}(O)) \in Q(T O)$ and $P(T O)^{-1}(F r(P(T O)(\operatorname{Int}(O))))$ $=\operatorname{Fr}\left(P(T O)^{-1}(P(T O)(\operatorname{Int}(O)))\right)[7]=\operatorname{Fr}(\operatorname{Int}(O))$ is compact, which implies $\operatorname{Fr}(P(T O)(\operatorname{Int}(O)))$ is compact [7]. Thus $\{P(T O)(\operatorname{Int}(O)) \mid O \in \mathcal{O}\}$ is a neighborhood base of $C$ consisting of neighborhoods with compact frontiers.

Conversely, suppose $(X(T O), Q(T O))$ is rim-compact. Let $x \in X$. Let $C \in X(T O)$ such that $x \in C$. Let $\mathcal{O}$ be a neighborhood base of $C$ consisting of neighborhoods with compact frontiers. Then $\{\operatorname{Int}(\mathcal{U}) \mid \mathcal{U} \in \mathcal{O}\}$ is a neighborhood base of $C$ consisting of neighborhoods with compact fronticrs and since for $\operatorname{cach} \mathcal{U} \in \mathcal{O}, \operatorname{Fr}\left(P(\operatorname{TO})^{-1}(\operatorname{Int}(\mathcal{U}))\right)=$ 
$P(T O)^{-1}(\operatorname{Fr}(\operatorname{Inl}(\mathcal{U})))$, which is compact [7], then $\left\{P(T O)^{-1}(\operatorname{Inl}(\mathcal{U})) \mid \mathcal{U} \in \mathcal{O}\right\}$ is a ncighborhood base of $x$ consisting of neighborhoods with compact fronticrs.

TIICOREM 16. Let $(X, T)$ be rim-compact. Then $(X, T)$ is 0 -dimensional iff $(X, T)$ is maximal disconnect.ed.

PROOF Suppose $(X, T)$ is 0-dimensional. Then $(X(T O), Q(T O))$ is rim-compact 0-dimensional $T_{1}$, which implies $(X(T O), Q(T O))$ is totally disconnected $[6]$ and thus $(X, T)$ is maximal disconnected

('onversely, suppose $(X, T)$ is maxımal disconnected Then $(X(T O), Q(T O))$ is rim-compact tolally disconnected $T_{1}$, which implics $(\mathrm{X}(T O), Q(T O))$ is 0 -dimensional [6] and thus $(X, T)$ is 0 -dimensional.

C'OROLLARY 17. Let $(X, T)$ be rim-compact. Then $(X, T)$ is 0 -dimensional $T_{0}$ iff $(X, T)$ is totally disconnected.

The last result in this section follows immediately from the fact that for $T_{1}$ spaces, metrizability and pscudometrizabılıty are equivalent

COROLLARY 18. Let $(X, T)$ be totally disconnected. Then $(X, T)$ is metrizable iff $(X, T)$ is pseudometrizable.

Thus in results known for totally disconnected metric spaces, metric can be replaced by pscudometric.

\section{REFERENCES}

1. KNASter, B. and KURATOWSKI, C. "Sur les ensembles connexes," Fund. Math, 2 (1921), 206-255.

2. SIFRPINSKI, W. "Sur les ensembles connexes et non connexes," Fund. Math., $\underline{2}$ (1921), 81-95.

3. DAVIS, $\Lambda$ "Indexed systems of ncighborhoods for general topological spaces," Amer Math. Monthly, $\underline{68}(1961)$, 886-893.

4. SIIANIN, N. "On separation in topological spaces," Dokl. Akad. Nauk.SSR, $\underline{38}$ (1943),110-113.

5. DORSETT, C. "Characterızations of spaces using $T_{0}$ - identification spaces," Kyungpook Math. J., 17, no. 2 (1977), 175-179.

6. WILlaRD, S. General Topology, Addison-Wesley Pub. Co., 1970.

7. DORSETT, C. " $T_{0}-$ identification spaces and $R_{1}$ spaces," Kungpook Math. J.,18, no. 2 (1978), 167-174.

8. "Strongly $R_{1}$ spaces," Kyungpook Math. J., 21, no. 2 (1981), 155-161.

9. DUNHAM, W. "Weakly Hausdorff spaces," Kyungpook Math. J.,15 (1975), 41-50. 


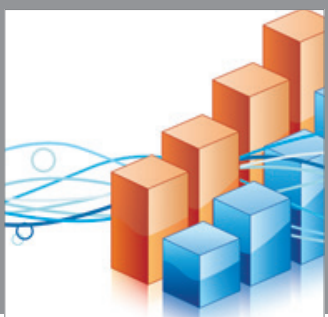

Advances in

Operations Research

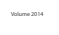

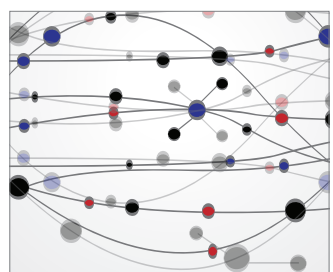

\section{The Scientific} World Journal
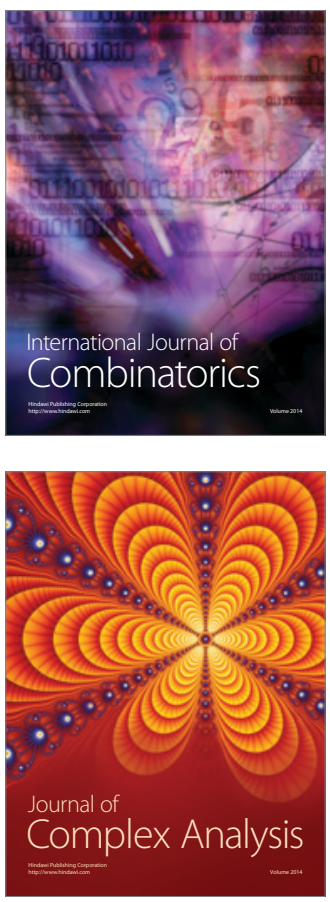

International Journal of

Mathematics and

Mathematical

Sciences
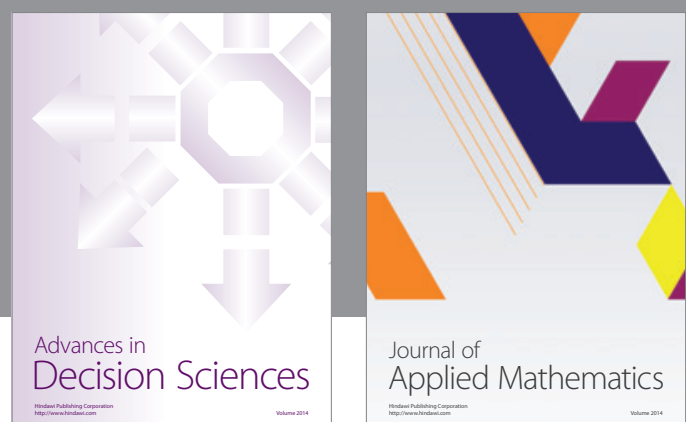

Journal of

Applied Mathematics
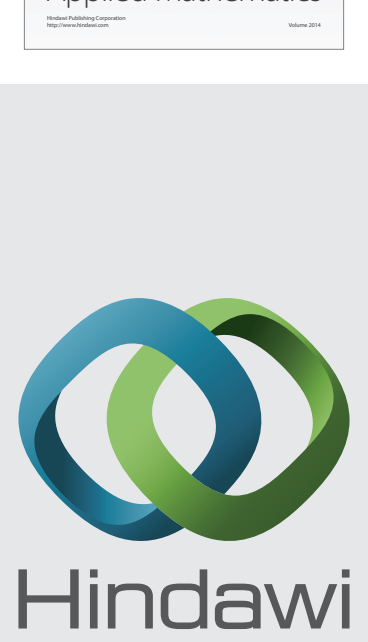

Submit your manuscripts at http://www.hindawi.com
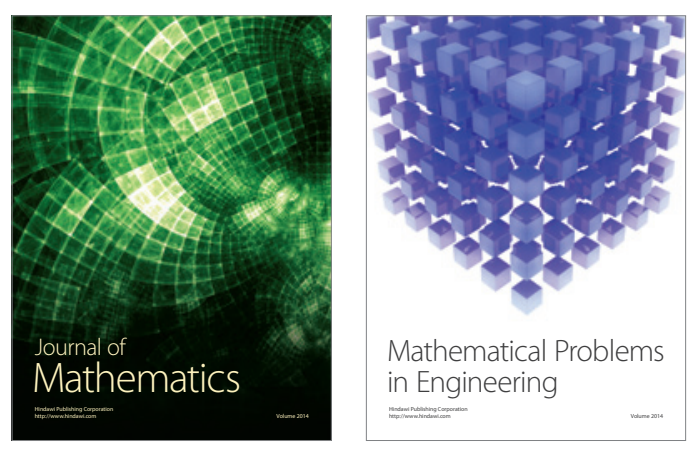

Mathematical Problems in Engineering
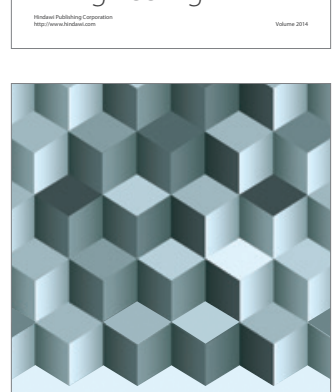

Journal of

Function Spaces
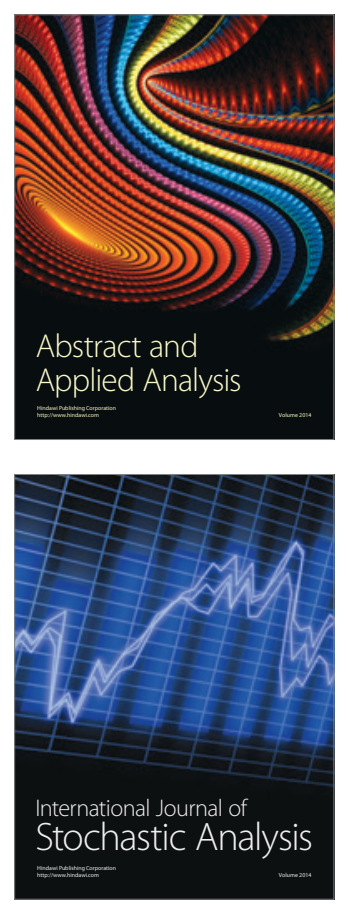

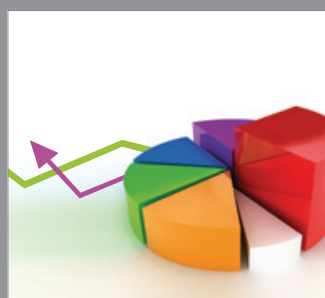

ournal of

Probability and Statistics

Promensencen
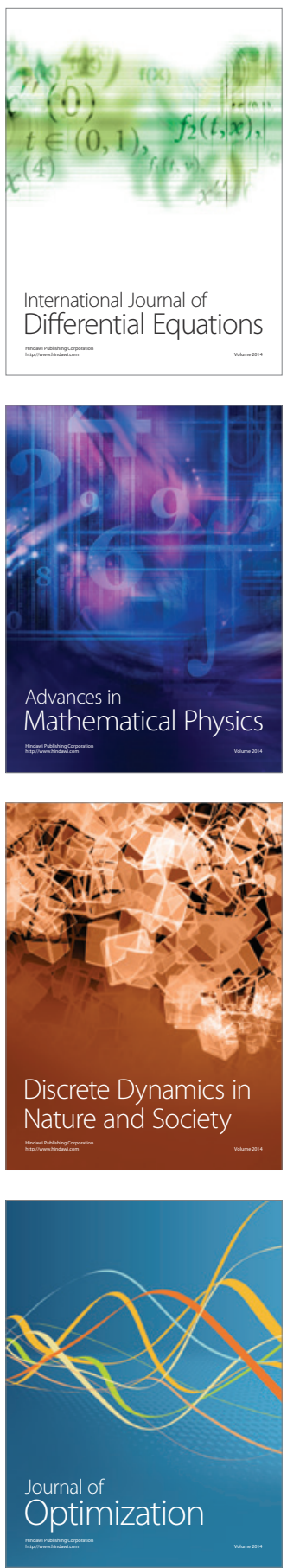